\title{
Multiples Juvenile Xanthogranuloma: About 4 Cases
}

\author{
Bouighjdane F*, Elaattoul N, Amal S, Hocar O
}

Department of Dermatology and Venereology, UHC Mohammed VI, FMPM, UCA, Marrakech

DOI: $10.36347 /$ sjmcr.2020.v08i01.014

| Received: 08.01.2020 | Accepted: 15.01.2020 | Published: 18.01.2020

*Corresponding author: Bouighjdane Fatiha

\section{Abstract}

Juvenile xanthogranuloma is a rare disease. It is a benign, auto-involutional tumor, mainly of cutaneous localization. We report 4 cases of multiple juvenile xanthogranuloma. There were 4 male children, whose average age was 1 year 5 months with extremes ranging from 3 months to 3 years. The average age of onset was 9 months. Lesions were multiple yellowish papulo nodules of varying size and number. The diagnosis of xanthogranuloma was confirmed on biopsy with histological and immunohistochemical study in all our patients. Neurofibromatosis type 1 was found in only one patient, in whom the hemogram showed a major hyperleucocytosis with hypochromic microcytic anemia and osteo-medullary biopsy infiltration by non-langerhansian histiocytic cells. Ophthalmological examination done in 3 patients returned normal. Abdominal ultrasonography revealed hepatosplenomegaly in one patient and was normal in the other 3. Juvenile xanthogranuloma is a form of non-langerhansian histiocytosis. It most often affects young children. Clinically occurs in its classic form as papules or nodules yellow orange firm and well limited. The anatomopathological examination confirms the diagnosis. Interrogation and clinical examination should focus on finding extra skin lesions and signs of Neurofibromatosis type1. Ophthalmological examination is justified in case of ocular signs, in case of extra-cutaneous lesions or multiple lesions in a child under 2 years old. If combined with NF1, a blood count should be made to search for associated myelomonocytic leukemia.

Keywords: Multiple xanthogranuloma, children, non-langerhansian, histiocytosis, neurofibromatosis.

Copyright @ 2020: This is an open-access article distributed under the terms of the Creative Commons Attribution license which permits unrestricted use, distribution, and reproduction in any medium for non-commercial use (NonCommercial, or CC-BY-NC) provided the original author and source are credited.

\section{INTRODUCTION}

Juvenile xanthogranuloma is the most common form of non-Langer histiocytosis. First described by Adamson in 1905, its current nomenclature was adopted in 1954. It is a benign auto-involutive tumor, mainly of cutaneous localization. We report 4 cases of juvenile muliple xanthogranuloma.

\section{Observations}

CASE 1: 9 month old infant from an unrelated marriage, follow-up for neurofibromatosis type 1, who was hospitalized for incomplete bone marrow failure syndrome (pallor, fever at $40^{\circ} \mathrm{C}$ ) and abdominal distension that had developed for 1 month before admission. The clinical examination found a tumor syndrome with a large splenomegaly, hepatomegaly and centimetric lymphadenopathy, and a skin syndrome associating 7 café-au-lait spots more than $5 \mathrm{~mm}$ in diameter, and yellowish papular lesions of 20 to $30 \mathrm{~mm}$, sitting on the trunk, back and scalp (Figures 1.2).

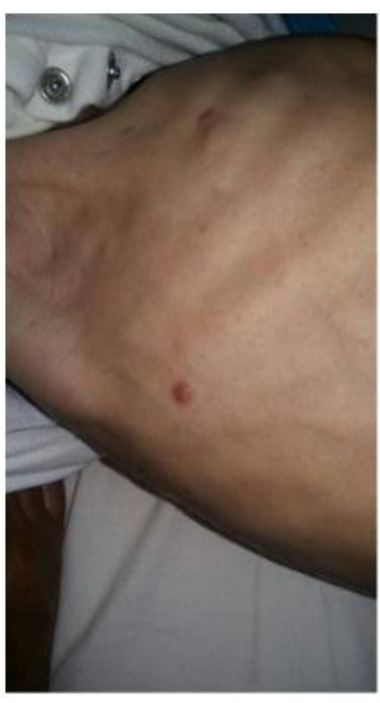

Fig-1: Orange-yellow nodule on the chest 


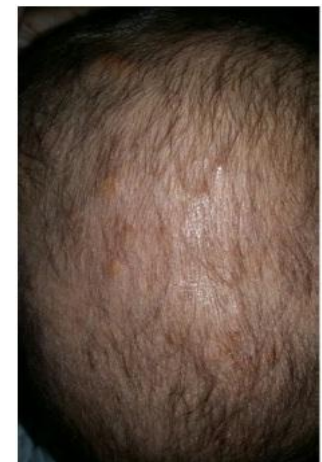

Fig-2: yellowish papules on the scalp

The hemogram showed major leukocytosis at $91,500 / \mathrm{mm} 3$, with monocytosis at $33,720 / \mathrm{mm} 3$, hypochromic microcytic anemia and normal platelet count. The myelogram did not show monocytic hyperplasia. The skin histology showed a dermal infiltration of non-langerhansian $\mathrm{CD} 68+, \mathrm{CD} 1 \mathrm{a}-$ histiocytic cells, leading to the diagnosis of juvenile xanthogranuloma. The osteo-medullary biopsy objectified a medullary infiltration by histiocytosis diagnosed on the skin. The ophthalmic examination was normal, as was the chest X-ray and renal doppler ultrasound. After CPR, the therapeutic decision was to repeat the myelogram after one month and monitor the patient.

CASE 2: Child of 3 and half years old, from a 1st degree related marriage, presented himself to the dermatology consultation for skin lesions evolving for 2 years. The clinical examination objectified firm yellowish papulo-nodules concerning the elbows and the fingers, with 2 ulcerative lesions in process of regression, one in the buttocks and the other in the elbow (Figures: 3.4 .5 ), absence of café au lait spots and tumor syndrome.

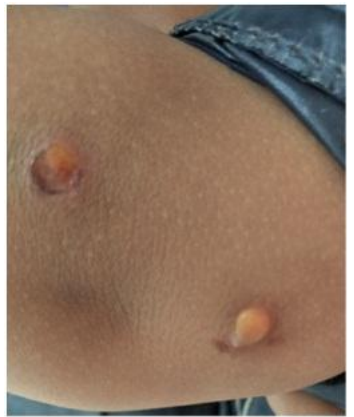

Fig-3: 2 yellowish nodules on the elbow, one of the nodules is ulcerated

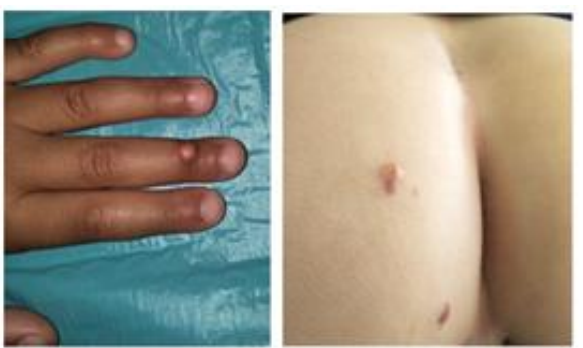

Fig-4, 5: yellow papules on the buttocks and fingers
The skin biopsy with anatomopathological study + IHC showed a morphological and immunohistochemical aspect compatible with a juvenile xanthogranuloma. The hemogram was normal and the abdomino-pelvic and renal ultrasound without abnormalities. The eye exam was unremarkable. Therapeutic abstention with monitoring every 3 months was the therapeutic decision.

CASE 3: 4-month-old infant without particular pathological ATCD who presented with 4 skin lesions on the chin, abdomen, and upper limbs. The clinical examination found firm nodular lesions of yellowish brown color, without café au lait spots, without hepatosplenomegaly and without lymphadenopathy. The anatomopathological study with IHC was in favor of a juvenile xanthogranuloma. The hemogram, chest $\mathrm{X}$-ray, and abdominal ultrasound were normal.

CASE 4: 20 month old infant presenting for 6 months with skin lesions without other associated signs. The clinical examination showed yellowish red papulonodules retro auricular, on the level of the testicles and the neck. The rest of the clinical examination was unremarkable. The histological study with IHC found an aspect in favor of a xanthogranuloma. The blood count, eye exam, abdominal ultrasound and chest X-ray were unrivaled.

\section{DisCUSSION}

Juvenile xanthogranuloma is the most common form of non-Langer histiocytosis [1-3]. Its etiology is unknown, some authors postulate the existence of an alteration of the macrophagic response to a nonspecific stimulus such as trauma and viral infection, a hypothesis that still lacks evidence.

Lesions appear during the first two years of life: 5 to $17 \%$ are present at birth and 40 to $70 \%$ during the first year [1.2]. Due to its mild and transient nature, it is estimated that it is a sub-entity.

The form with multiple lesions represents 7 to $10 \%$ of cases of juvenile xanthogranuloma and it is characterized by a male predominance and this is the case for our patients, with onset before the age of 6 months [3.4].

Clinically presented in its classic form as firm, well-limited orange-yellow papules or nodules. [3], Some telangiectasias may be present in area. It is generally asymptomatic but he can ulcerate himself. It is unique in more than $60 \%$ of cases, but multiple or even disseminated forms are reported. The preferred areas are the head and neck, the trunk and limbs [4].

The extra cutaneous involvement in juvenile xanthogranuloma is dominated by ocular involvement, followed by pulmonary, then hepatic, splenic, pancreatic and renal involvement [4,5]. 
The ocular involvement is represented by a hyphema, glaucoma, uveitis, an iris heterochromia [4,5]. The ophthalmological examination is justified in the event of signs of ocular appeal and in children less than 2 years of age with multiple lesions [5].

Classically described are associations with type 1 neurofibromatosis, juvenile chronic myeloid leukemia, and acute lymphoblastic leukemia and with myelomonocytic leukemias. The concomitant presence of juvenile xanthogranuloma and NF type 1 brings an increased risk (20 to 30 times) of developing juvenile chronic myeloid leukemia [6, 7]. In our small series, only one child had neurofibromatosis type 1 and the same patient had abnormal hemograms but the myelogram was normal.

Histopathology shows typical histiocytosis findings, with frequent infiltration of the dermis, presence of multinucleated giant cells in variable numbers, and inflammatory cells in the perilesional areas. In $85 \%$ of cases, it is possible to observe Touton giant cells, resulting from the fusion of macrophages and characterized by a crown of nuclei, with homogeneous eosinophilic cytoplasmic center and prominent peripheral xanthomatization[1,4, 8-10].

In immunohistochemical terms, xanthogranuloma histiocytes do not express markers of Langerhansian histiocytosis, CD1 and protein S. They express Macrophagic markers, vimentin and CD68 [1, 8-10].

The prognosis of patients with exclusively cutaneous involvement is excellent, with spontaneous remission in months or a few years, and relapses are rare. In some cases, a small residual hyperpigmented scar may remain. The cases with extracutaneous involvement present greater morbidity and mortality [4].

Considering the benign and self-involutive character, a therapeutic abstention is the rule. Surgical excision is offered for diagnostic or aesthetic reasons $[3,4]$.

In case of ocular involvement, corticosteroid treatment is indicated and in case of failure, radiotherapy is an alternative [3, 4]. In the event of visceral involvement with repercussions on the functions vital treatment based on chemotherapy, radiotherapy, corticosteroid therapy or cyclosporine can be used $[3,4]$.

\section{CONCLUSiON}

Juvenile xanthogranuloma is generally mild with a regression in children in three at six years according to the data in the literature. The associated ophthalmological involvement is the severity of the disease. In the presence of multiple lesions, in children under two years of age, an ophthalmologic examination is indicated every six months up to two years. The clinical examination and the interrogation look for elements that can guide towards the diagnosis of NF1. Additional examinations in search of visceral localizations are requested according to the signs of clinical orientation. The appearance of multiple juvenile xanthogranuloma in a child with NF1 prompts a biopsy to confirm the diagnosis. The risk of an LMMJC incites to carry out a close clinical surveillance in search of hepatosplenomegaly, lymphadenopathy and a monitoring of the blood count in the search for a monocytosis.

\section{REREFENCES}

1. Weitzman S, Jaffe R. Uncommon histiocytic disorders: The non-Langerhans cell histiocytoses. Pediatric Blood \& Cancer. 2005;45(3):256-64.

2. Rana A, de Waal Malefyt S. An Infant Who Has Dome-Shaped Papules. Pediatrics in Review. 2012;33(2):86-8.

3. Szczerkowska-Dobosz A, Kozicka D, PurzyckaBogdan D. Juvenile xanthogranuloma: a rare benign histiocytic disorder. Advances in Dermatology and Allergology. 2014;3:197-200.

4. Kupfer-Bessaguet I. Xanthogranulomejuvénile. Annales de Dermatologie et de Vénéréologie. 2009;136(1):69.

5. Hamdani M, El Kettani A, Rais L. Juvenile xanthogranuloma with intraocular involvement. A case report. J Fr Ophtalmol. 2000;23(8):817-20.

6. Fenot M, Stalder J-F, Barbarot S. Juvenile xanthogranulomas are highly prevalent but transient in young children with neurofibromatosis type 1. Journal of the American Academy of Dermatology. 2014;71(2):389-90.

7. Zvulunov A. Juvenile Xanthogranuloma, Neurofibromatosis, and Juvenile Chronic Myelogenous Leukemia: World Statistical Analysis. Archives of Dermatology. 1995;131(8):904.

8. Pajaziti L, Hapçiu SR, Pajaziti A. Juvenile xanthogranuloma: a case report and review of the literature. BMC Research Notes. 2014;7(1).

9. Dehner LP. Juvenile Xanthogranulomas in the First Two Decades of Life: A Clinicopathologic Study of 174 Cases with Cutaneous and Extracutaneous Manifestations: The American Journal of Surgical Pathology. 2003;27(5):579-93.

10. Chang MW. Update on juvenile xanthogranuloma: Unusual cutaneous and systemic variants. Seminars in Cutaneous Medicine and Surgery. 1999;18(3):195-205. 